\title{
APLICACIÓN DE NUEVOS MÉTODOS DIDÁCTICOS EN EL AULA PARA ASIGNATURAS DE COMPUTACIÓN: UTILIZACIÓN DE UN SOFTWARE CLIENTE / SERVIDOR
}

\author{
Juan José Leiva Olivencia ${ }^{1}$ \\ José Luis Leiva Olivencia ${ }^{2}$
}

\begin{abstract}
Resumen: En el presente trabajo queremos exponer de forma breve los aspectos metodológicos de un método didáctico que estamos utilizando actualmente en algunas asignaturas de computación, basado en el uso de software cliente / servidor, en el que aplicar las nuevas tecnologías de la información y la comunicación (NTIC). Este método, llevamos experimentándolo en nuestro centro en diversas asignaturas de Informática (Educación Secundaria, Bachillerato y Universitaria), pero consideramos que puede ser muy viable su utilización en otro tipo de materia, no solo en las de Informática, sino que sus posibilidades pueden ser ampliadas como recurso didáctico en otras asignaturas o materias del curriculum en estos mismos niveles educativos.
\end{abstract}

El software que nosotros utilizamos, realmente se compone de dos programas que se irán ejecutando simultáneamente: el módulo o software del profesor, que estará instalado únicamente en el ordenador del profesor y que podemos denominar aplicación servidor y el software de los alumnos, que denominamos aplicación cliente, el cual deberá instalarse en todos los equipos informáticos que vayan a utilizar los alumnos. En este sentido, si bien en el mercado existen diferentes aplicaciones que son similares a la que nosotros utilizamos, nuestro trabajo pretende ser una aportación innovadora acerca de nuestra experiencia práctica sobre las mismas, en lo que sería la integración de las TICs en la docencia.

Palabras claves: SOFTWARE SERVIDOR / CLIENTE. NUEVAS TECNOLOGÍAS DE LA INFORMACIÓN. AULA INTERACTIVA. APLICACIÓN INFORMÁTICA. COMUNICACIÓN INTERACTIVA.

\begin{abstract}
We are going to expose brief the methodological aspects of a didactic method based on software Client/ Servant in that to apply the new technologies of the information and the communication (NTIC). This method we go it experimenting on our center on diverse subjects of Computer science (Secondary Education and High Secondary Education), but we think that it can be very viable his utilization in another type of matter, not only in those of Computer science, but his possibilities can be extended as didactic resource in other subjects or matters of the curriculum in the same educational levels. The software that we use, really $i$ consists of two modules: the module of the teacher, which only will establish itself in the computer of the teacher and that we prune names an application servant and the module of the pupils, that we name application client, who will have to establish in all the computer equipment's that are going to use the pupils. In this respect, though on the market there exist different applications that are similar to that we use, our work tries to be an innovative contribution brings over of our practical experience on the same ones, in what it would be the integration of TICS in the teaching.
\end{abstract}

Keywords: SOFTWARE SERVANT / CLIENT. NEW TECHNOLOGIES OF THE INFORMATION. INTERACTIVE CLASSROOM. COMPUTER APPLICATION. INTERACTIVE COMMUNICATION.

\footnotetext{
${ }^{1}$ Profesor del Departamento de Teoría e Historia de la Educación, Universidad de Málaga. España. e-mail: ijleivac@uma.es

2 Profesor del Departamento de Lenguajes y Ciencias de la Computación. Universidad de Málaga, España.

e-mail: illeivao@/cc.uma.es
}

Artículo recibido: 6 de octubre, 2003

Aprobado: 24 de noviembre, 2003 


\section{Introducción}

Las aulas equipadas con ordenadores conectados en red de área local, se han convertido hoy en día en un recurso muy habitual en las escuelas, y que poco a poco se van haciendo casi imprescindible. No obstante, siendo un recurso muy importante (acceso a Internet mediante proxy, compartir recursos, entre otros), es cierto que también es una fuente de preocupación en los docentes, pues es un recurso de gran potencial didáctico y pedagógico, por lo que las estrategias didácticas deben ser canalizadas de forma planificada para indagar en procesos de aprendizaje de calidad.

En este sentido, si bien desde la perspectiva de los docentes que imparten clases en materias tales como informática $u$ otras asignaturas relacionadas con las tecnologías de la información, en las que la utilización de equipos informáticos, Internet y otras herramientas tecnológicas resultan fundamentales, y partiendo por tanto de la gran ventaja que supone su utilización en los alumnos por el valor intrínsecamente atractivo y motivador de los mismos; no es menos cierto que tenemos la experiencia de que cuando enseñamos a los alumnos a trabajar alguna aplicación específica , es muy común que se distraigan en ese espacio tan atractivo, perdiéndose en algún mensaje, menú, o en otras acciones que pueda posibilitar la aplicación determinada.

En definitiva, el aula de PC es un recurso ambivalente, pues ofrece claras ventajas, pero a su vez es un importante elemento perturbador de la clase, ya que al acaparar la atención del alumno, lo aleja del profesor que no tiene facilidades para aprovechar el importante recurso del que dispone.

En efecto, el aprendizaje de una herramienta software implica un proceso continuo y puntual de todos sus aspectos por parte del alumno en su aprendizaje significativo, y ciertamente no tiene ya sentido intentar explicar el manejo de estas herramientas por medio de las tradicionales trasparencias o bien en el encerado, etc. En realidad, creemos que se necesita, por tanto, un método que permita la interacción del alumno en su aprendizaje con el objeto del mismo, esto es, mostrarles el proceso correcto de cómo se resuelven los problemas sin que el alumno pueda perderse en otros aspectos, mediante su participación activa en la resolución de los mismos y su implicación progresiva en el marco de un aprendizaje cada vez más autónomo y responsable. Ciertamente, las implicaciones de un 
aprendizaje autónomo en el manejo de las herramientas informáticas constituye un eje clave en la necesaria comprensión, no sólo de los propios contenidos de la materia, sino también, de las formas de aprender, interactivas y dinámicas.

\section{2. ¿Cómo funciona, en términos técnicos, nuestro método docente? Elementos sobre los que interactuar.}

El método que nosotros utilizamos, permite trabajar con los siguientes elementos:
a) Permite realizar visitas guiadas de páginas de Internet.
b) Permite mostrar el contenido de la pantalla del profesor en el resto de pantallas, o en un grupo, o de un solo alumno.
c) Permite bloquear ordenadores (teclado y ratón).
d) Permite ejecutar aplicaciones en el ordenador del alumno.
e) Permite apagar y reiniciar el ordenador del alumno.
f) Enviar mensajes.
g) Enviar archivos.

Existen varios tipos de software similar al que utilizamos nosotros, pero con una importante laguna, y es que nosotros adoptamos los roles de profesor y alumno, los cuáles "encaminados" a las necesidades del aula, permiten obtener un mayor aprovechamiento en relación a la orientación del aprendizaje de los alumnos en la consecución de los objetivos curriculares.

Por otro lado, si hacemos un breve repaso sobre los distintos métodos educativos, nos encontramos dos grandes familias, los métodos afirmativos y los de elaboración:

a) Métodos afirmativos: se basan en transmitir los conocimientos sin una participación activa del alumno. Son principalmente comunicaciones expositivas (clase magistral) o métodos demostrativos, mediante el cual el alumno visualiza un trabajo práctico realizado por el profesor. Por tanto, podemos admitir que la actitud del alumno es típicamente receptiva, estática y pasiva. Además, este tipo de metodología no reconoce al alumno como agente activo - y comprometido - en su aprendizaje, por lo que se le niega su participación, y también, el reconocimiento de su responsabilidad en su autonomía como estudiante. 
b) Métodos por elaboración: el profesor y los alumnos intervienen conjuntamente de forma activa en la realización de una tarea. El alumno se convierte en sujeto y verbo, al tiempo que objeto de su propia formación. En realidad, estamos haciendo referencia a todas aquellas estrategias que giran en torno a las propias iniciativas de los alumnos, que conjuntamente con las propuestas del docente, indagan en actividades concretas y consensuadas, fruto del diálogo y la cooperación entre los propios alumnos y con el profesor.

Nosotros nos proponemos maximizar las ventajas potenciales de las nuevas tecnologías, utilizando el modelo de Rockart (1979), en el que se contemplan los siguientes estados: adquisición, asimilación, integración y verificación. En todos estos aspectos, el elemento esencial que deben trabajar los alumnos es la simulación, hecho básico en nuestra experiencia. Este modelo tiene como elemento transversal el desarrollo de tareas ordenadas y dirigidas en el aprendizaje del alumno, como punto en el que subyacen los diferentes estados anteriormente señalados. En verdad, nos referimos a los momentos claves en el desarrollo de las estrategias de aprendizaje en su interacción con las tareas orientadas por el profesor en el aula. Este tipo de planteamientos se enmarca en un contexto determinado por las orientaciones teóricas y metodológicas del aprendizaje relevante y significativo señaladas por Escudero (1992), que hace referencia a la ineludible necesidad de integrar las nuevas tecnologías de la información y las comunicaciones en los planteamientos pedagógicos y didácticos de los docentes de las escuelas actuales, insertadas, como todos sabemos, en la sociedad del conocimiento. Esta sociedad se caracteriza por la necesidad de generar y transmitir el conocimiento como forma primordial de desarrollar nuevos recursos $y$ materiales, para que, a partir de su experimentación y difusión, puedan generar a su vez nuevas necesidades para la promoción de nuevos conocimientos tecnológicos. El ser humano se encuentra en una encrucijada tecnológica en la que debe saber comprender sus mecanismos y condicionantes, para ser un ciudadano activo y responsable en esta nueva sociedad del conocimiento, globalizada tanto económica como culturalmente. En este sentido, nuestra experiencia profesional nos lleva a contextualizar nuestro método interactivo en el marco de la extraordinaria capacidad de las TICs como elementos de aprendizaje necesarios para la formación integral de nuestro alumnado, así como de la urgente necesidad de profundizar en nuevas propuestas didácticas y pedagógicas que nos ayuden como docentes a mejorar nuestra práctica y desarrollo profesional. 
Cuando se estudia Informática en la escuela, hay que entender que se adquieren unas especiales características (Escudero, 1992). Por un lado, al principio surge la lógica curiosidad de una asignatura que tiene unas particularidades respecto a otras, que a veces hace que existan más esperanzas de las que debiera. Inevitablemente, la informática está muy relacionada con el mundo de los juegos, las videoconsolas, Internet, entre otros; y por tanto la con la diversión y el entretenimiento. Esto hace que, luego a la hora de la verdad, cuando aparecen conceptos tales como 'bases de datos', 'lenguajes de programación', etc; puede llegar a hacer de la Informática un final desdichado, desmotivando o no cumpliendo con las iniciales expectativas de los alumnos. Por tanto, hay que intentar mezclar dos conceptos de igual forma: diversión y aplicación de los conceptos de forma precisa y clara, para hacer que la informática sea una herramienta de aprendizaje dinámica y relevante.

Por tanto, una faceta a "explotar" una vez pasada la curiosidad, sería explorar la informática como una materia fascinante, pero a su vez como una materia, que por regla general ejercerá en el futuro laboral de los alumnos una amplia influencia. Ni que decir tiene, que estamos en una sociedad del conocimiento y de la información, y hoy en día resulta absolutamente imprescindible tener las capacidades y las competencias para acceder a la información y al conocimiento, porque son el motor del desarrollo social y económico de nuestras sociedades. De ahí, la importancia de la informática como pieza clave en este nuevo panorama.

\section{Actividades básicas en el aula.}

Vamos a exponer un ejemplo que permita clarificar nuestro propósito de indagar en un método interactivo en el aula. Supongamos por ejemplo que se está trabajando con un procesador de textos. Así pues, a todos los alumnos les indicamos que se descarguen desde la página del profesor en Internet un archivo con un documento Word; el propósito de esta tarea es la de explicarles el proceso de inserción de gráficos y objetos en un texto determinado.

Una vez que todos los alumnos tienen en su ordenador su documento, la aplicación servidor hará que en el monitor de todos los alumnos aparezca el contenido del monitor del profesor, no pudiendo por tanto el alumno realizar acción alguna en su ordenador. 
En este punto, el profesor pasará a explicar desde su ordenador los conceptos fundamentales relacionados con el tema en cuestión, realizando los pasos necesarios en su ordenador. Así pues, el alumnado podrá observar en su monitor como el profesor realiza las tareas. En efecto, este método evita la distracción con otros elementos del ordenador, ya que en ese momento el programa cliente acapara todos los recursos del ordenador del alumno.

No obstante, una vez explicados los conceptos claves del trabajo propuesto, el alumno toma de nuevo el control de su ordenador, teniendo que realizar ahora en su documento, las tareas realizadas por el profesor. En caso de duda, lógicamente el profesor se acercará a resolverlas de forma individual o en pequeño grupo, pero en el caso de que un grupo mayor de alumnos tengan la misma duda, la aplicación servidor puede tomar de nuevo el control de los ordenadores de aquellos alumnos que tienen problemas en el cumplimiento de la tarea, permitiendo resolverla simultáneamente a varios alumnos.

Desde luego, esta herramienta permite también la comunicación mediante mensajes desde el profesor al alumno y viceversa. Además, otra característica importante de este método consiste en la propia posibilidad de que el profesor, desde su ordenador, tome el control del ordenador del alumno, e incluso que varios alumnos puedan ver como el profesor interactúa con el ordenador de un compañero.

Otra ventaja de este tipo de aplicaciones, es la posibilidad de hacer guiada y orientativa, la visita a páginas web en Internet (Gallego y Alonso, 1999), ya que el profesor al indicar una dirección determinada, hace que los navegadores de los alumnos visiten dicha página, imposibilitando la visita a otras páginas no previstas en el desarrollo del trabajo propuesto en clase, en tanto la barra dirección se bloquea e imposibilita la distracción de los alumnos.

En efecto, nuestro propósito es el de encauzar el aprendizaje del alumno en estos momentos de enseñanza tutorizada, ya que en posteriores sesiones, el alumno podrá ejercer mayor autonomía en sus actividades. Se trata, por tanto, de iniciar un modelo interactivo que resulte eficaz en el proceso de enseñanza y aprendizaje, sin menoscabar, en ningún caso, las posibilidades y las iniciativas de los alumnos, ya que estos podrán desarrollar nuevas actividades con el consenso y acuerdo conjunto entre ellos mismos y el profesor, de manera 
que, si inicialmente, el proceso está dirigido, está claro que las posibilidades de desarrollo de este modelo interactivo son mayores.

\section{Roles de participación del alumno.}

El alumno, al participar en este proceso, puede tomar varios papeles, siendo en todos ellos un elemento vital, en el que su participación enriquecerá las clases. Así, distinguimos tres papeles: el de alumno, el de profesor, y el de miembro de un grupo.

\section{1. Alumno como emisor.}

Lógicamente el papel de alumno no tiene mucha explicación, pero en determinadas situaciones el alumno puede hacer el papel o rol de profesor, que se trata de un aspecto interesante que contempla nuestra propuesta, mediante la cual el propio alumno, al llegar a una conclusión de alguna tarea que pueda ser interesante su resolución para el resto de los compañeros, puede mostrar su solución al resto de los compañeros de forma sencilla.

El ordenador del alumno, que se trata de un equipo cliente, se convierte en un equipo servidor, siendo ahora sus compañeros los que reciben la clase por parte de el. Se trata de un aspecto altamente motivador, ya que el propio alumno es capaz de desarrollar la resolución a sus compañeros, permitiendo a veces llegar a sus compañeros de una forma más directa que el profesor, que a veces utiliza un lenguaje demasiado técnico, etc. Ni que decir tiene, que esta dinámica supone un paso relevante y significativo en el marco de un método interactivo.

\subsection{El alumno en un grupo de trabajo.}

Un aspecto muy importante en la enseñanza, es el trabajo en equipo y colaborativo. Además, siendo un tema tan crucial, todos sabemos que es ciertamente difícil conseguir que los alumnos trabajen eficientemente en equipo, porque nos están acostumbrados a este tipo de dinámica. Con nuestra metodología, mediante la cual los alumnos pueden intercambiar información y recursos, fomentamos el trabajo cooperativo.

Ejemplos que muestren el trabajo en equipo, pueden ser la ayuda que se prestan entre ellos mediante el paso de mensajes, o permitiendo a un alumno hacer las funciones de profesor, con lo que el compañero ve en su ordenador cómo hacer alguna determinada tarea, etc. 
A pesar, de que puede ser tentador que algunos alumnos no hagan nada y que se aprovechen de otros compañeros que trabajan, debemos hacer notar que nuestra experiencia nos dice que el trabajo del grupo es en general mucho más eficiente que en otros cursos donde no hemos utilizado esta metodología. En este sentido, una cuestión clave, es la propia dinámica de aprendizaje cooperativo, que trasciende la propia enseñanza de informática, pero que constituye un eje crucial en nuestro método interactivo. En efecto, debemos ser conscientes de las posibilidades del aprendizaje compartido y grupal, ya que todos aprendemos de todos, por lo que debemos ser capaces de indagar en dinámicas que generen una conciencia colectiva de comprensión de la cooperación como clave en el aprendizaje, no sólo individual, de cada uno de los alumnos, sino también del conjunto de ellos, como grupo-clase, y como colectivo en general de jóvenes interesados en aprender (Gómez y Sáenz del Castillo, 2000).

\section{Características tecnológicas.}

Los ordenadores son capaces de enviar información en paquetes IP a un grupo de ordenadores en lugar de uno solo de una forma bastante eficiente. Esto se conoce con el nombre de multidifusión. Esta es la base del método. Las redes de área local utilizar un medio de transmisión compartido, por lo que los mensajes que se propagan por la red 'los ven' pasar todas las máquinas. Esta es por tanto la idea central del método, hacer que el ordenador del profesor pueda enviar el contenido de su pantalla, mensajes, archivos, etc. A todos los ordenadores de los alumnos a la vez.

Podría pensarse que si el número de alumnos es muy grande, el tráfico en la red aumentará, pero no es cierto, ya que cuesta lo mismo enviar un mensaje a un solo ordenador, que a todos, sea cual sea el número de ordenadores conectados en la red, aunque si los cambios de pantallas son muchos entonces el tráfico aumenta considerablemente.

También queremos hacer constar que la aplicación cliente se ejecuta directamente al encender el ordenador, y al alumno no le aparecerá en la barra de tareas, para hacerlo así invisible al alumno. Otra de las características es la imposibilidad de cerrar la aplicación por parte del alumno de forma manual, pues el control de ejecución no es manejable por el alumno. 
Parece evidente, que los requisitos mínimos necesarios es disponer de una red de área local, y en el caso de visita guiada por Internet, conexión a Internet y Proxy. Esto son elementos básicos, pero fundamentales para llevar a cabo un tipo de metodología interactiva, que facilite y refuerce la comunicación entre profesor y alumno, así como la amplia gama de posibilidades que entraña, en relación a las posibles oportunidades de aprendizaje cooperativo entre los propios alumnos, siempre y cuando contribuya a su aprendizaje significativo y relevante.

De todas formas los requisitos mínimos que consideramos necesarios para poner en práctica nuestro método son:

- Procesador Pentium II $400 \mathrm{MHZ}$ o superior.

- Tarjeta video $4 \mathrm{MB}$ o superior.

- Disco Durso 2GB o superior.

- Tarjeta de red 10/100 Mbps

- CD-ROM

- Sistema Operativo Windows 98 o superior

- Protocolo TCP/IP 


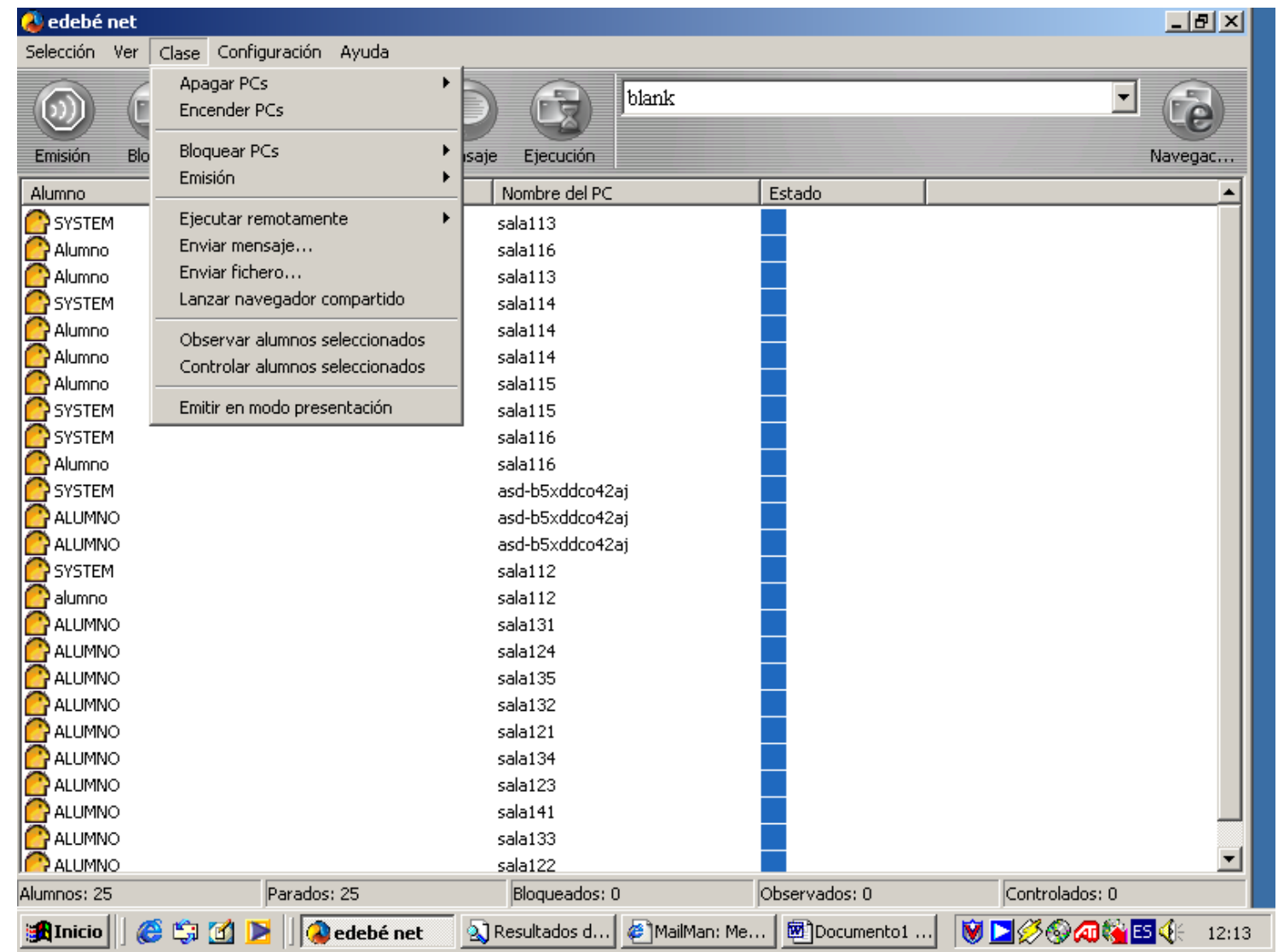

También queremos hacer constar que a los alumnos se les ha quitado permiso para poder copiar archivos en equipos distintos al suyo, siendo previamente necesario que el profesor active un permiso para aquellos casos necesarios, con esto pretendemos fomentar el trabajo y la comunicación, y evitar así, actitudes individualistas que no se encaminen hacia el aprendizaje compartido y el diálogo entre ellos mismos. Se trata, por tanto, de una opción que haga imprescindible la comunicación interactiva, para comprender este tipo de metodología en su propio sentido y contexto de enseñanza y aprendizaje (González, 1983).

\section{El profesor y su competencia profesional.}

Desde luego una de las claves más importantes en el planteamiento didáctico que hemos desarrollado hasta ahora tiene que ver con las capacidades y competencias del propio docente a la hora de llevar a cabo un modelo interactivo de enseñanza y aprendizaje en las tareas educativas que se pueden establecer en el área de informática. Esto nos lleva a pensar sobre aquellos conocimientos que a nivel global debe tener un docente para integrar en su aula la informática y la navegación por Internet como recursos didácticos fundamentales, algo que ya hemos mencionado anteriormente, pero que dada su 
extraordinaria importancia insistimos en ello, añadiendo que no sólo tiene especial relevancia para las asignaturas y materias propias de informática, sino que sistemas interactivos como el que proponemos pueden ser sumamente eficaces al extender este tipo de iniciativas a otros ámbitos disciplinares.

En este punto, tal y como plantean Gallego y Alonso (1999), es primordial cuestionarnos acerca de los conocimientos que debe poseer un docente para integrar en su aula la Informática y la navegación por Internet, de ahí que consideremos adecuado exponer las directrices de la Asociación Internacional de Informática Educativa (ISTE), que propone una serie de habilidades de gran interés y que sirven de base para diseñar un programa de aprendizaje para los docentes, algo fundamental para poder planificar, promover y desarrollar sistemas didácticos apoyados en la computación. Son las siguientes:

1. Demostrar capacidad para manejar un sistema informático con el fin de utilizar bien el software.

2. Evaluar y utilizar el ordenador y la tecnología asociada a él para apoyar el proceso educativo.

3. Aplicar los principios educativos actuales, las investigaciones y los ejercicios de evaluación al uso informático y a las tecnologías asociadas a él.

4. Explorar, evaluar y utilizar el material informático/tecnológico, incluidas las aplicaciones, el software educativo y la documentación asociada.

5. Demostrar conocimiento de los usos del ordenador para la resolución de problemas, recolección de datos, gestión de la información, comunicaciones, presentación de trabajos y toma de decisiones.

6. Diseñar y desarrollar actividades de aprendizaje que integren la informática y la tecnología para estrategias de grupos de alumnos y para las diversas poblaciones de estudiantes.

7. Evaluar, seleccionar e integrar la enseñanza mediante la informática en el curriculum del área temática y/o nivel educativo correspondiente.

8. Demostrar conocimiento del uso del multimedia, hipermedia y telecomunicaciones para favorecer los procesos de enseñanza y aprendizaje.

9. Demostrar habilidad en el empleo de herramientas de productividad para uso personal y profesional, incluidos el procesador de textos, la base de datos, la hoja de cálculo y las utilidades de impresión y gráficos. 
10. Demostrar conocimiento de los problemas de equidad, éticos, legales y humanos, relacionados con el uso de la informática y la tecnología, en cuanto éste sé relaciona con la sociedad y contribuye a conformar las conductas sociales y culturales.

11. Identificar los recursos para mantenerse al ida en el aprendizaje de las nuevas aplicaciones informáticas y las tecnologías afines en el campo educativo.

12. Utilizar las tecnologías informáticas para acceder a la información que incremente la productividad personal y profesional.

13. Aplicar los ordenadores y las tecnologías afines para favorecer las nuevas funciones del educando y el educador (formación en entornos virtuales y aprendizaje on-line...)

\section{Conclusiones}

En este trabajo hemos descrito de forma resumida la aplicación de nuevas tecnologías a la mejora de la calidad docente. Nuestra experiencia se enmarca en la puesta en práctica de este método en la asignatura de Informática en los diferentes cursos de E.S.O., Bachillerato y algunas asignatura universitarias, pero todas de Informática. Pensamos que puede resultar muy valioso y de gran utilidad para asignaturas tales como Historia (visitas guiadas por Internet), Biología e Idiomas, por ejemplo. Además, este método interactivo elimina problemas sencillos y cotidianos que pueden ocurrir con la utilización de otros instrumentos como las trasparencias o la explicación en encerado, en tanto hace que los alumnos permanezcan atentos a sus pantallas y a sus equipos informáticos, en la línea de un aprendizaje cada vez más individualizado y global, atendiendo en su caso, a las oportunidades que nos ofrecen estas herramientas tecnológicas para el aprendizaje cooperativo de los alumnos, que también indagan en las posibilidades de un aprendizaje personal que les facilite también su desarrollo y progreso en otras materias.

En una sociedad como la actual, sometida a tan profundos y acelerados cambios, las funciones y los comportamientos del profesor tienen que experimentar alteraciones significativas. Sin menospreciar el componente "artesanal" de la tarea docente, resulta evidente que la tecnificación de los procesos educativos es un hecho irreversible. La educación se apoya cada vez más en principios científicos y técnicos, tendiendo a identificarse con un modelo tecnológico de información y comunicación. La propia escuela, en su configuración actual y futura, tiene que hacer frente al doble reto de un cuestionamiento crítico sobre su función y posibilidades y la incorporación, en su práctica 
institucional, de las sugestiones de los medios de comunicación social y las nuevas tecnologías.

\section{Referencias}

ESCUDERO, J. M. (1992). La integración escolar en las nuevas tecnologías de la información. Infodidac 21,11-24.

GALLEGO, D.J. Y ALONSO, C.M. (1999) Multimedia en la Web. Madrid: Dykinson.

GÓMEZ GALÁN, J. y SÁENZ DEL CASTILLO, A. A. (2000). Nuevas Tecnologías Aplicadas a la Educación. Badajoz: Universitas Editorial-I.C.E. de la Universidad de Extremadura.

GONZÁLEZ, M. (1983). Enseñanza asistida por ordenador. Madrid: Anaya.

ROCKART, J. F. (1979). Chief executive define their own data needs. Harvard Bussiness Review, March-April. 